\title{
Effect of strong coupling on interfacial electron transfer dynamics in dye-sensitized $\mathrm{TiO}_{2}$ semiconductor nanoparticles
}

\author{
HIRENDRA N GHOSH \\ Radiation \& Photochemistry Division, Bhabha Atomic Research Centre, Trombay, Mumbai 400085 \\ e-mail: hnghosh@barc.gov.in
}

\begin{abstract}
Dynamics of interfacial electron transfer (ET) in ruthenium polypyridyl complex $\left[\left\{\right.\right.$ bis- $\left(2,2^{\prime}-\right.$ bpy)-(4-[2-(4'-methyl-[2,2']bipyridinyl-4-yl)-vinyl]-benzene-1,2-diol) \}ruthenium(II) hexafluorophosphate] (Ru-cat) and 5,10,15-tris phenyl-20-(3,4-dihydroxy benzene) porphyrin (TPP-cat)-sensitized $\mathrm{TiO}_{2}$ nanoparticles have been investigated using femtosecond transient absorption spectroscopic detection in the visible and near-infrared region. We have observed that both Ru-cat and TPP-cat are coupled strongly with the $\mathrm{TiO}_{2}$ nanoparticles through their pendant catechol moieties. We have observed a single exponential and pulse-width limited (<100 fs) electron injection from nonthermalized-excited states of Ru-complex. Here electron injection competes with the singlet-triplet manifold relaxation due to strong coupling of catecholate binding, which is a unique observation.

Optical absorption measurements indicate that the catechol moiety interacts with $\mathrm{TiO}_{2}$ nanoparticles showing the characteristic pure catechol- $\mathrm{TiO}_{2}$ charge-transfer (CT) band in the visible region. Transient absorption studies on TPP-cat/ $/ \mathrm{TiO}_{2}$ system exciting both the Soret band at $400 \mathrm{~nm}$ and the Q-band at $800 \mathrm{~nm}$ have been carried out to determine excitation wavelength-dependence on ET dynamics. The reaction channel for the electron-injection process has been found to be different for both the excitation wavelengths. Excitation at $800 \mathrm{~nm}$, is found directly populate directly the excited CT state from where diffusion of electrons into the conduction band takes place. On the other hand, excitation at $400 \mathrm{~nm}$ light excites both the CT band of cat- $\mathrm{TiO}_{2}$ and also Soret band of TPP-cat.
\end{abstract}

Keywords. Interfacial electron transfer dynamics; $\mathrm{TiO}_{2}$ semiconductor nanoparticles; transient absorption spectroscopy.

\section{Introduction}

Relentless efforts are underway all over the world to understand the dye sensitization of wide-band gap semiconductor nanomaterials (e.g. $\mathrm{TiO}_{2}, \mathrm{SnO}_{2}, \mathrm{ZnO}$ ) in view of their potential technological implication in photovoltaic energy. ${ }^{1}$ In this area, maximum effort has been focused on dye molecules based on metal polypyridine complexes and their analogues. ${ }^{2,3}$ So far, tris bipyridyl ruthenium (II) dyes have been regarded as the best sensitizing dyes for solar energy conversion for their strong visible absorption bands, long-lived excited states and excellent photochemical stability. However, the development of organic sensitizers, which can exhibit performances similar to those of metal complexes, gained a lot of interest in the context of dye-sensitized solar cells. During the last few years, a number of organic dyes, such as phthalocyanines, tri-phenyl methane, xanthenes, coumarins, and porphyrins have been tested as sensitizers, but the light-to-electricity conversion efficiency is rather low compared to what has been shown by metal polypyridine complexes. ${ }^{3}$ However, the metal polypyridine complexes show poor red/near-infrared light absorption. In view of the rich photochemical and electrochemical properties of porphyrins, the low cost of production and their broad absorption in the visible and NIR region, many researchers have taken keen interest in elucidating the factors, which limit their efficiency in solar cells based on dye-sensitized nanocrystalline $\mathrm{TiO}_{2}$. High affinity for the $\mathrm{TiO}_{2}$ surface, which is provided by the anchoring groups of the sensitizer molecules, is one of the important parameters. In most of the studies the anchoring groups of the dye molecules used in dye-sensitized solar cells including those polypyridine complexes and porphyrins are carboxylate, which bind the dye molecules with the nanoparticles. However, there are certain disadvantages in using carboxylates as anchoring groups. The ground state $\mathrm{p} K_{a}$ of the carboxylates is too low to ensure strong binding. ${ }^{4}$ Also, slow desorption of the photosensitizers can occur in the presence of water which may limit the long-term stability of the 
cell. We and various other groups have observed that catecholate binding is stronger than any other type of binding. Polypyridyl complexes with the pendant catechol moiety show considerable promise in the context of realizing the phenomenon of electron injection from non-thermalized states of the dye molecule. It has been suggested theoretically by Rego et $a l^{5}$ that, contrary to the direct charge transfer excitation in $\mathrm{TiO}_{2}$-catechol systems, efficient photo-injection mechanisms can involve electronic excited states in the molecular adsorbate in the $\mathrm{Ru}(\mathrm{II})$-polypyridyl-catecholate. Catechol binding with $\mathrm{TiO}_{2}$ nanoparticles through a five-membered chelate formation is expected to be thermodynamically very stable. In our previous studies we have shown that pyrogallol red (PGR), ${ }^{6}$ and alizarin $^{7}$ molecules bind strongly with $\mathrm{TiO}_{2}$ nanoparticles through the catechol moiety and have large electronic coupling with $\mathrm{TiO}_{2}$. In the present article we have discussed interfacial electron transfer dynamics of $\mathrm{Ru}(\mathrm{II})$-polypyridyl-catecholate and porphyrin catechol with $\mathrm{TiO}_{2}$ nanoparticles also to understand the influence of electronic coupling on ET dynamics using ultrafast femtosecond absorption spectroscopy.

\section{Experimental}

\subsection{Materials}

Titanium(IV) tetraisopropoxide $\left\{\mathrm{Ti}\left[\mathrm{OCH}\left(\mathrm{CH}_{3}\right)_{2}\right]_{4}\right\}$ (Aldrich, 97\%), and isopropyl alcohol (Aldrich) were purified by distillation. Nanopure water (Barnsted System, USA) was used for making aqueous solutions; $\quad\{$ bis-(2,2'-bpy)-(4-[2-(4'-methyl-[2,2']bipyridinyl-4-yl)-vinyl]benzene-1,2-diol) $\}$ ruthenium(II) hexafluorophosphate ((Ru-cat) was synthesized by adopting reported procedure ${ }^{8}$ with some modification in the purification procedure. Synthesis of 5,10,15-tris phenyl-20-(3,4-dihydroxy benzene) porphyrin (TPP-cat) is reported elsewhere. ${ }^{9}$

\subsection{Sample preparation}

Nanometre-size $\mathrm{TiO}_{2}$ was prepared by controlled hydrolysis of titanium (IV) isopropoxide and it has been described in detail in our earlier work. ${ }^{6,7}$

\subsection{Femtosecond visible spectrometer}

The femtosecond tunable visible spectrometer (figure 1) has been developed based on a multi-pass amplified femtosecond Ti:sapphire laser system from Avesta, Russia $(1 \mathrm{kHz}$ repetition rate at $800 \mathrm{~nm}$, $50 \mathrm{fs}, 800 \mu \mathrm{J} /$ pulse) and described earlier. ${ }^{7}$ The $800 \mathrm{~nm}$ output pulse from the multipass amplifier is split into two parts to generate pump and probe pulses. In the present investigation, we have used both $800 \mathrm{~nm}$ (fundamental) and it's frequency doubled $400 \mathrm{~nm}$ as excitation sources. To generate pump pulses at $400 \mathrm{~nm}$, one part of $800 \mathrm{~nm}$ with $200 \mu \mathrm{J} /$ pulse, is frequency doubled in BBO crystals. To generate visible probe pulses, about $3 \mu \mathrm{J}$ of the $800 \mathrm{~nm}$ beam is focused onto a $1.5 \mathrm{~mm}$ thick sapphire window. The intensity of the $800 \mathrm{~nm}$ beam is adjusted by iris size and ND filters to obtain a stable white light continuum in the $400 \mathrm{~nm}$ to over $1000 \mathrm{~nm}$ region. The probe pulses are split into the signal and reference beams and are detected by two matched photodiodes with variable gain. We have kept the spot sizes of the pump beam and probe beam at the crossing point around 500 and $300 \mathrm{mi}-$ cron respectively. The excitation energy density (at both $800 \mathrm{~nm}$ and $400 \mathrm{~nm}$ ) was adjusted to $\sim 2500 \mu \mathrm{J} / \mathrm{cm}^{2}$. The noise level of the white light is about $\sim 0.5 \%$ with occasional spikes due to oscillator fluctuation. We have noticed that most laser noise is low-frequency noise and can be eliminated by comparing the adjacent probe laser pulses (pump blocked vs unblocked using a mechanical chopper). The typical noise in the measured absorbance change is about $<0.3 \%$. The instrument response function was obtained by fitting the rise time of the

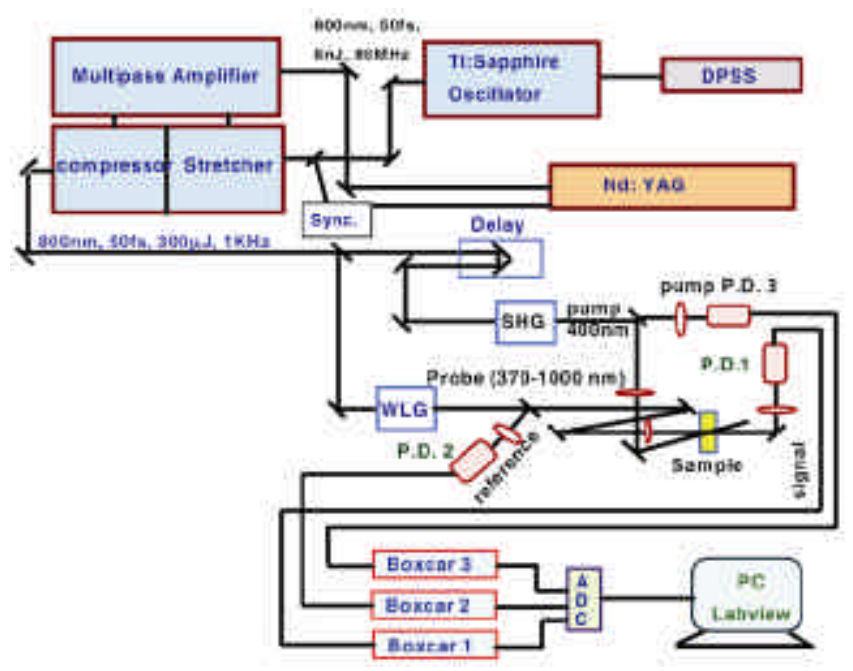

Figure 1. Molecular structure of Ru-cat and TPP-cat. Mode of interaction with $\mathrm{TiO}_{2}$ nanoparticles through the catecholate moiety. 
bleach of sodium salt of meso tetrakis (4 sulphonatophenyl) porphyrin (TPPS) at $710 \mathrm{~nm}$, which has an instantaneous response.

\subsection{Cyclic voltammetry}

Electrochemical experiments were performed on a $\mathrm{CH}-$ 660A (USA) electrochemical instrument; a conventional three-electrode cell assembly was used. Oxidation redox potential of Ru-cat and TPP-cat molecule has been determined to be $1.32 \mathrm{~V}$ and $+0.79 \mathrm{~V}$ against $\mathrm{Ag} / \mathrm{AgCl}$ electrode respectively.

\section{Results and discussion}

\subsection{Dye-nanoparticle interaction}

The nature of the binding between a sensitizer and a semiconductor directly influences the excited-state and interfacial ET behaviour. ${ }^{10}$ Strong binding serves to anchor the sensitizer in place, control interfacial electronic coupling, and tune the redox potentials of the sensitizers and the semiconductor. However, the affinity of catecholate dye molecules towards $\mathrm{TiO}_{2}$ semiconductor nanoparticles is better as compared to the carboxylate or phosphate dye molecules, which can establish better linkage than the other two. Optical absorption studies emphasized that Ru-cat and TPP-cat interact strongly with $\mathrm{TiO}_{2}$ nanoparticles ${ }^{11}$ with the formation of a five-membered complex as shown in figure 2. Optical absorption spectrum of TPP-cat shows a Soret band with a maximum at $435 \mathrm{~nm}$ and a main Q-band with a maximum at $665 \mathrm{~nm}$ (figure 3). Steady state optical absorption measurements shows that on addition of
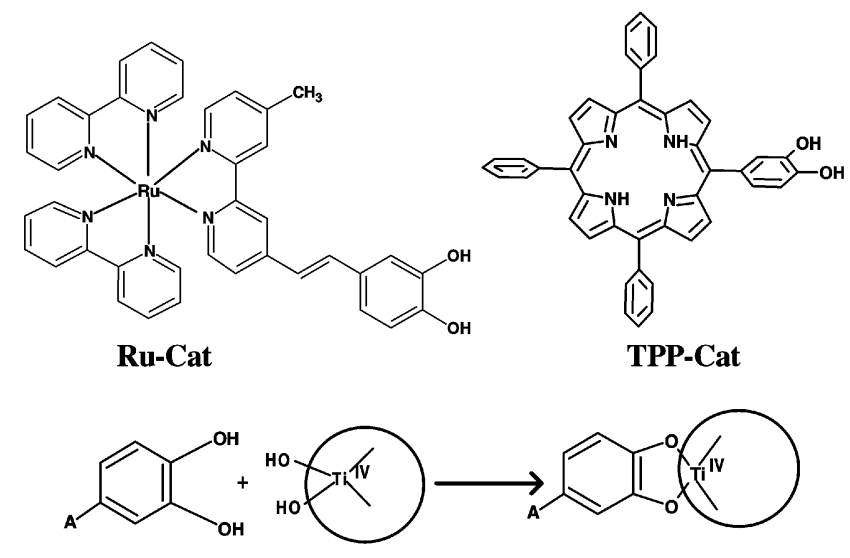

Figure 2. Schematic diagram of femtosecond transient absorption spectrometer.
$\mathrm{TiO}_{2}$ nanoparticles, the absorption spectrum of the Q-band of TPP-cat becomes broad and shifts to longer wavelengths with increase in absorbance for the $Q$ band at $\approx 700 \mathrm{~nm}$. Red shift $\approx 40 \mathrm{~nm}(660 \mathrm{~nm}$ to $700 \mathrm{~nm}$ ) and broadening of Q band of TPP-cat in presence of $\mathrm{TiO}_{2}$ nanoparticles has been attributed to the formation of a charge transfer (CT) complex. ${ }^{12}$ It is very interesting to observe that the Soret band of TPP-cat does not change much in presence of $\mathrm{TiO}_{2}$ nanoparticles, however there is an increment of optical absorption in the $400-600 \mathrm{~nm}$ region. It that a new band appears at $400-600 \mathrm{~nm}$ region on top of the original Soret band. In this article we have shown that TPP-cat also interacts strongly with $\mathrm{TiO}_{2}$ nanoparticles with the formation of a five-membered $\mathrm{CT}$ complex as shown in figure 1 . From our measurements it is quite clear that TPP-cat molecules interact strongly with $\mathrm{TiO}_{2}$ nanoparticles with the formation of two different types of CT complex. One CT complex (complex 1) is due to interaction of the catechol moiety and $\mathrm{TiO}_{2}$ absorbing in 400$600 \mathrm{~nm}$ region and to the other CT complex (complex 2) is due to the Q-band of TPP-cat interacting with $\mathrm{TiO}_{2}$ absorbing at the $650-900 \mathrm{~nm}$ region.

\subsection{Steady-state emission studies in $\mathrm{TPP}$-cat/TiO 2 system}

We have also carried out emission measurements of freebase TPP-cat and its protonated form, as shown in figures $4 \mathrm{a}$ and $\mathrm{b}$ respectively. Freebase TPP-cat

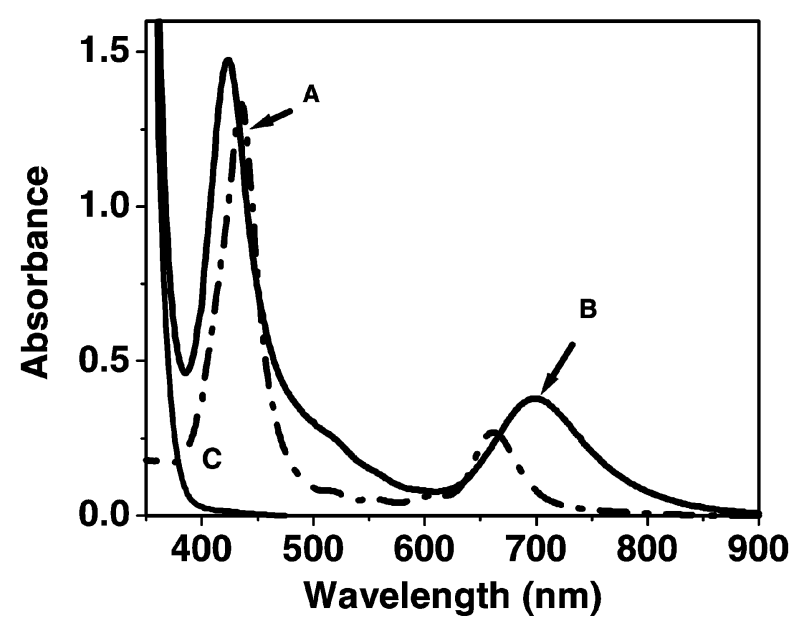

Figure 3. Optical absorption spectrum of TPP-cat in water (A), on $\mathrm{TiO}_{2}$ nanoparticle $(10 \mathrm{gm} / \mathrm{L})$ surface (B) and the absorption spectrum of $\mathrm{TiO}_{2}$ nanoparticle $(10 \mathrm{gm} / \mathrm{L})$ in water $(\mathrm{C})$. 
emits at $655 \mathrm{~nm}$ with a shoulder at $718 \mathrm{~nm}$ (figure 3a). However, the protonated form emits at single wavelength at $676 \mathrm{~nm}$ (figure 3b), which is located between the two emission bands (originally observed before adding acid). Our results agree well with the observations made by Akins et $a l^{47}$ for the case of TPP with the addition of tri-fluoro acetic acid. We have carried out steady-state emission measurements of TPP-cat both in the presence and absence of $\mathrm{TiO}_{2}$ nanoparticles. It is observed in figure $4 \mathrm{c}$ that in the presence of $\mathrm{TiO}_{2}$ nanoparticles the emission intensity decreases drastically. This is due to electron injection into the $\mathrm{TiO}_{2}$ from the photoexcited TPP-cat molecule. However, to understand the detailed electron transfer dynamics in the TPPcat $/ \mathrm{TiO}_{2}$ system, we have carried out ultrafast transient absorption spectroscopy.

\subsection{ET dynamics in $\mathrm{Ru}$-cat/TiO $\mathrm{O}_{2}$ system}

We have carried out transient absorption experiments for Ru-cat-sensitized $\mathrm{TiO}_{2}$ nanoparticles excited at $400 \mathrm{~nm}$, to follow the interfacial ET dynamics on the semiconductor surface. Figure 5 shows the transient absorption spectra of Ru-cat-sensitized $\mathrm{TiO}_{2}$ nanoparticles at different time delay. The spectrum at each time delay consists of bleach in 470 $500 \mathrm{~nm}$ wavelength region with a maximum at $\approx 480 \mathrm{~nm}$, an absorption peak at $\approx 590 \mathrm{~nm}$ and another broad positive absorption band in the $700-1000 \mathrm{~nm}$ region which has been attributed to injected electrons in the conduction band. ${ }^{6,7}$ The ab-

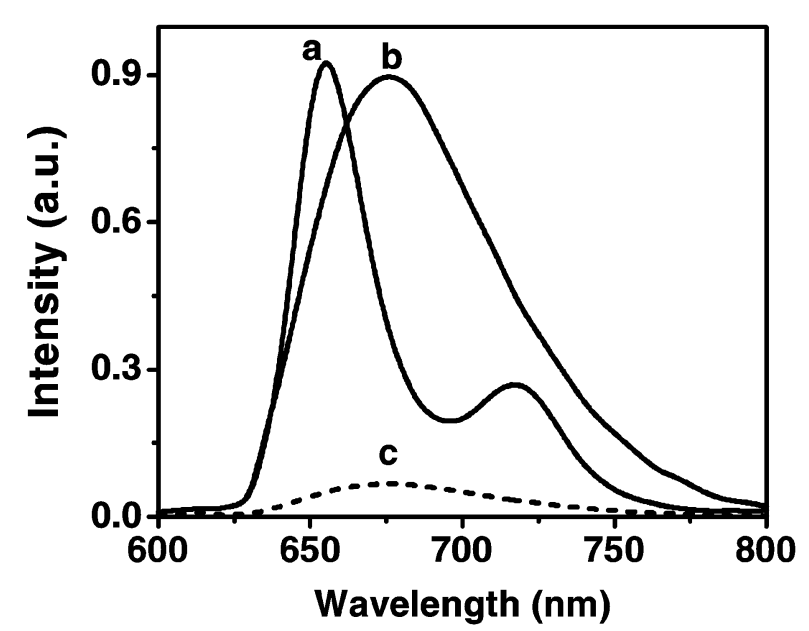

Figure 4. Steady-state emission spectra of TPP-cat in water at pH 6 (a) and $\mathrm{pH} 2.5$ (b) and on $\mathrm{TiO}_{2}$ nanoparticle $(10 \mathrm{gm} / \mathrm{L})$ surface $(\mathrm{c})$. sorption peak at $590 \mathrm{~nm}$ has been assigned to the Ru-cat cation radical. ${ }^{11}$ Injection time in the present investigation has been determined by monitoring the appearance time of the signal of the injected electron. Figure 6 inset shows the kinetic trace of the injected electron at $900 \mathrm{~nm}$. The injection time has been found to be pulse-width limited ( $<100 \mathrm{fs})$ and single exponential. It has been observed that the kinetic decay trace follows a multi-exponential function with time constants of $1.5 \mathrm{ps}(34.8 \%), 70 \mathrm{ps}$ $(26.8 \%)$ and $400 \mathrm{ps}(38.4 \%)$ that can be attributed to back electron transfer dynamics.

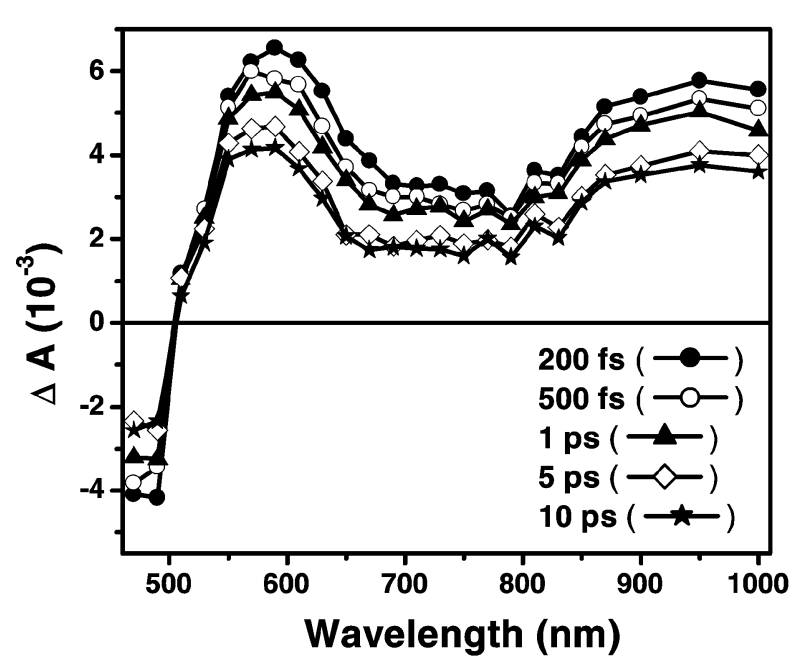

Figure 5. Transient absorption spectra of Ru-cat sensitized $\mathrm{TiO}_{2}$ nanoparticles in water at different time delays after excitation at $400 \mathrm{~nm}$.

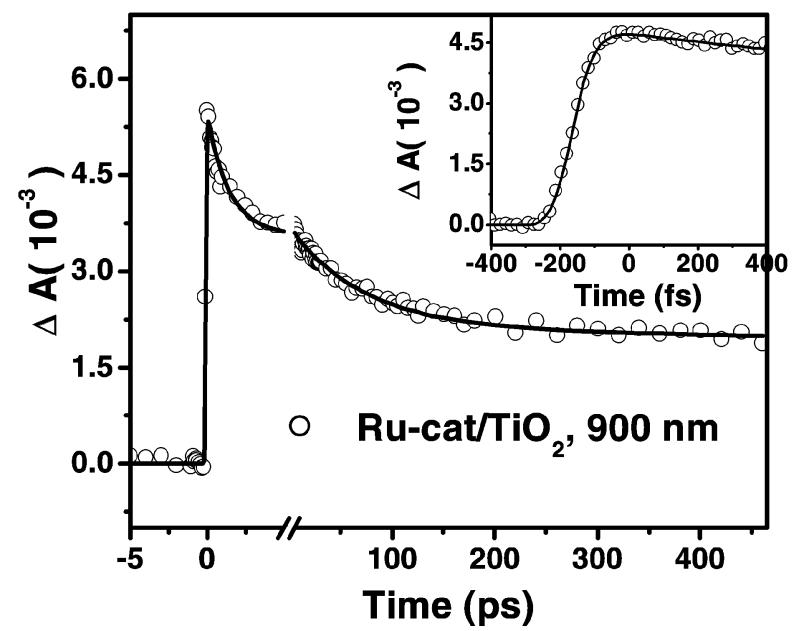

Figure 6. Kinetic decay trace of injected electrons in the conduction band at $900 \mathrm{~nm}$ in $\mathrm{Ru}$-cat sensitized $\mathrm{TiO}_{2}$ nanoparticles (inset shows the kinetic trace at $900 \mathrm{~nm}$ at shorter time scale). 
Charge-transfer dynamics in Ru-(dcbpy $)_{2}(\mathrm{NCS})_{2}$ (widely known as $\mathrm{Ru}-\mathrm{N} 3$ ) sensitized $\mathrm{TiO}_{2}$ nanomaterials has been a subject of intense research interest for the last ten years. Much of the recent work has focused on quantifying the rate of excited-state electron injection into the semiconductor. ${ }^{13,14}$ In the above system, different electron-injection rates have been reported. It has been shown by femtosecond transient near IR spectroscopy that at least in some cases ${ }^{13}$ injection is ultrafast and occurs prior to vibrational relaxation of the thermally equilibrated MLCT manifold, i.e., from "hot" excited states or the Frank-Condon state itself. However, most of the authors agree on the presence of sub-100 fs component and consider it to be the dominant channel with additional contributions of slower non-exponential electron injection with a distribution of characteristic times from $1 \mathrm{ps}$ to tens of picoseconds. The origin of discrepancy of different electron injection dynamics is still under debate. In most of the studies it has been observed that injection dynamics is ultrafast and biphasic. Two assumptions have been made to explain the origin of the lack of single exponentiality: (i) because of the inhomogeneity of the semiconductor surface, the adsorbed dye molecules have different geometric configurations resulting different electronic coupling elements, giving rise to different rates, (ii) the fast and slow components are due to electron injection from non-thermalized ${ }^{1}$ MLCT and thermalized ${ }^{3}$ MLCT states, respectively. However, most of researchers believe in a second hypothesis, where competition between ET from the non-thermalized states and ISC to triplet states, vibrational cooling or thermalization of triplet states is seen. Recently Bhasikuttan et $a l^{15}$ have observed that ISC process of N3 dye can be as fast as $<50 \mathrm{fs}$. So it can really compete with the electron-injection process. It has been clearly demonstrated by Benko et $a l^{14}$ that electron injection in $\mathrm{N} 3 / \mathrm{TiO}_{2}$ system occurs mainly from the non-thermalized ${ }^{1}$ MLCT state with a smaller contribution from the ${ }^{3}$ MLCT state. Most of the researchers prefer to attribute bi-phasic injection by the second mechanism as suggested above i.e., injection from ${ }^{1}$ MLCT and ${ }^{3}$ MLCT rather than the inhomogeneities of the semiconductor surface.

One of the central points of this investigation is whether it is possible at all to observe single exponential electron injection from the photo-excited poly-pyridyl dyes to the conduction band of $\mathrm{TiO}_{2}$ nanoparticles. The answer is affirmative, provided it has to fulfill certain conditions. Lian and co-workers have smartly demonstrated that if the ${ }^{3}$ MLCT state of an adsorbate lies substantially below the conduction band edge then we can suppress the slow component; as a result the electron injection will be dominated by the fast component. They have observed this phenomenon in Re-carbonyl complexes (ReCO) on dry $\mathrm{TiO}_{2}$ film, ${ }^{13}$ where ReCO's relaxed excited state is estimated to be $-0.35 \mathrm{~V}$ (SCE) lower than that of $\mathrm{TiO}_{2}$ dry film $(-0.5 \mathrm{~V}$ at $\mathrm{pH} 2)$. However, in the present investigation, the relaxed ${ }^{3}$ MLCT state of Ru-cat lies above the conduction band edge (scheme 1). So, in principle it cannot fulfill the condition, which has been demonstrated by Lian and co-workers in $\mathrm{ReCO}$ complexes on $\mathrm{TiO}_{2}$ film. On the other hand, in our earlier studies, we have observed single exponential electron injection time constant in many organic dye-nanoparticle systems,${ }^{6,7}$ where electron injection would only take place from the singlet excited state since the singlettriplet ISC takes long time (>1 ns). In such systems strong electronic coupling of the dye molecules with the nanoparticles would govern the electron-injection dynamics. Strong coupling facilitates ultrafast electron injection from excited singlet state before it relaxes to the relaxed state. In the present investigation, we have observed that injection dynamics is single exponential and pulse-width limited (inset of

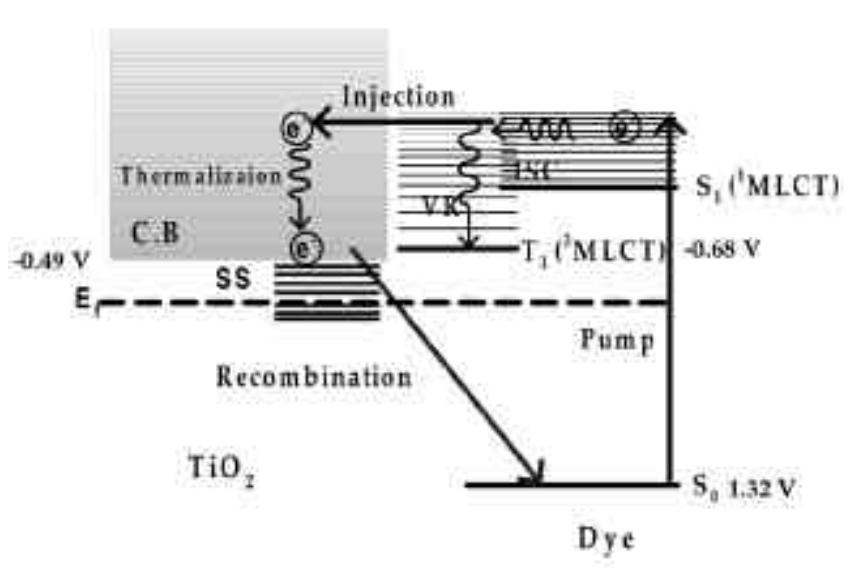

\section{V.B}

Scheme 1. Mechanistic scheme showing three-level model which consists of the ground state $\left(S_{0}\right)$ state, the excited triplet state $\left({ }^{3} \mathrm{MLCT}\right)$ state and the excited singlet $\left({ }^{1} \mathrm{MLCT}\right)$ state of $\mathrm{Ru}(\mathrm{II})$-polypyridyl catecholate adsorbed on $\mathrm{TiO}_{2}$ nanoparticles. 
figure 6). Even though Ru-cat complex can have both singlet $\left({ }^{1}\right.$ MLCT) and triplet $\left({ }^{3}\right.$ MLCT) manifold in the excited states, still we have observed single component in the injection kinetics. Although, it was reported earlier ${ }^{14}$ that in the Ru-polypyridyl$\mathrm{TiO}_{2}$ system electron injection from ${ }^{1}$ MLCT (singlet) state competes with the ISC process to the ${ }^{3}$ MLCT (triplet) state. In the present investigation due to very fast ISC process, non-thermalized excited states can be due to both non- thermalized ${ }^{1}$ MLCT (singlet) or non-thermalized ${ }^{3}$ MLCT (triplet) states or a combination of both hot singlet and triplet states (scheme 1). As a consequence of large electronic coupling in the present $\mathrm{Ru}-\mathrm{cat} / \mathrm{TiO}{ }_{2}$ system, electron injection takes place predominantly from non-thermalized states (scheme 1) rather than from thermalized states. It is reported in the literature ${ }^{5}$ that on excitation of the MLCT band of Ru-polypyridyl complexes, the electron gets excited from the $\mathrm{Ru}$ metal centre and localized in the bi-pyridyl ligands. So it is quite obvious that on excitation with $400 \mathrm{~nm}$ laser light, the electron from the $\mathrm{Ru}(\mathrm{II})$ gets localized in one of the bi-pyridyl (bpy) ligands and also in the bpy-cat ligand. As bpy-cat is directly and strongly coupled with the $\mathrm{TiO}_{2}$ nanoparticles, injection process takes place from bpy-cat ligand $\left(\mathrm{TiO}_{2}\right.$ surface-attached ligand). On the other hand, the other two bpys are not attached directly to the nanoparticle so the de-excitation process from singlet to triplet manifold is possible. However, the redox potential of bpy-cat ligand is lower $(-1.09 \mathrm{~V})$ as compared to other bpy ligands $(-1.19 \mathrm{~V}$ and $-1.59 \mathrm{~V}),{ }^{16}$ as a result upon excitation, photo-excited electron from ruthenium metal ion prefers to get localized mostly at the bpy-cat ligand, from where fast electron injection will take place due to direct coupling with the nanoparticles ${ }^{11}$. As the localization takes place mostly on bpy-cat ligand, ILET process does not interfere much in the electron injection process at least for the present system. As a consequence, we have observed single exponential and pulsewidth limited electron injection kinetics. On the other hand, it is very interesting to observe that recombination dynamics in $\mathrm{Ru}-\mathrm{cat} / \mathrm{TiO}_{2}$ system is faster as compared to other Ru-poly-pyridyl complex (like N3) sensitized $\mathrm{TiO}_{2}$ nanoparticles systems. ${ }^{13} \mathrm{In}$ carboxylate anchored Ru-polypyridyl complex sensitized $\mathrm{TiO}_{2}$ system, coupling strength for ET reaction is much lower as compared to that in catecholate group anchored complex. As we have observed the strong binding of the catecholate group with nanoparticles helps ultrafast single exponential injection. Similarly, it has been observed that stronger binding of catecholate group also influences the BET dynamics, making it faster.

\subsection{ET dynamics in $\mathrm{TPP}$-cat/TiO ${ }_{2}$ system}

One of the central themes of the present article is to understand the interfacial ET dynamics of TPPcat $/ \mathrm{TiO}_{2}$ system excited at $400 \mathrm{~nm}$ where the Soret band and CT band of catechol/ $/ \mathrm{TiO}_{2}$ complex (complex 1) absorb, and excited at $800 \mathrm{~nm}$ where the CT band of complex 2 due to interaction of Q-band and $\mathrm{TiO}_{2}$ nanoparticles absorbs (figure 3). It is observed in figure 3 that both the CT complex of catechol/ $/ \mathrm{TiO}_{2}$ and the Soret band of TPP-cat absorb at $400 \mathrm{~nm}$. As a result, with $400 \mathrm{~nm}$ laser light we can excite both the complex 1 and the Soret band of porphyrin molecule. Figure 7 shows that the transient spectrum at each time delay consists of an absorption peak at $\approx 610 \mathrm{~nm}$, bleach in $640-750 \mathrm{~nm}$ wavelength region with a maximum at $\approx 700 \mathrm{~nm}$, and another broad positive absorption band in 760 $1000 \mathrm{~nm}$ region which is attributed to the conduction band electron. The absorption peak at $610 \mathrm{~nm}$ has been assigned to TPP-cat cation radical. ${ }^{17}$ Electron injection time $\left(\tau_{\text {inj }}\right)$ has been determined by monitoring the appearance signal of the conduction band electron at $900 \mathrm{~nm}$ (Figure 8). We have observed that $\mathrm{BET}$ dynamics in $\mathrm{TPP}$-cat $/ \mathrm{TiO}_{2}$ is very fast

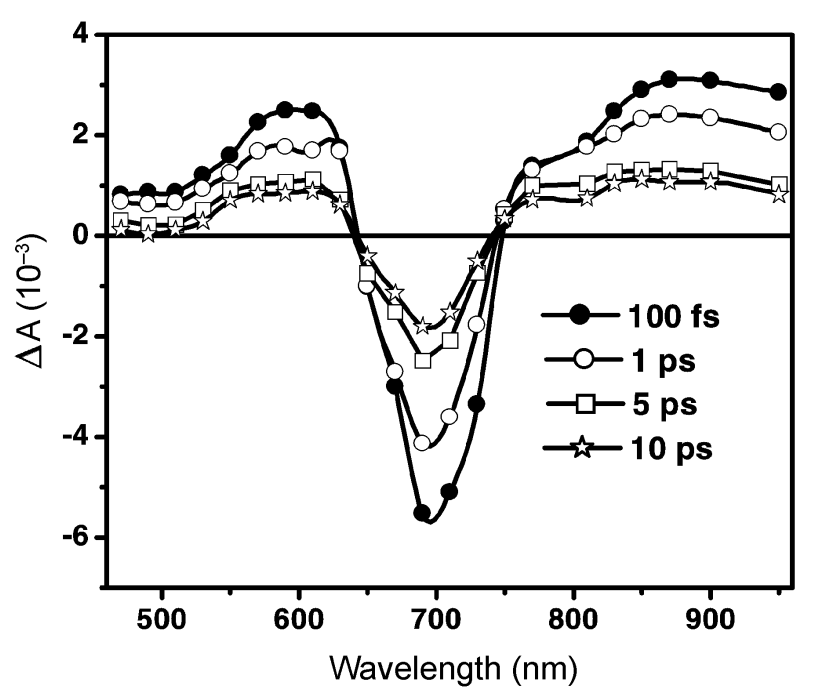

Figure 7. Transient absorption spectra of TPP-cat sensitized $\mathrm{TiO}_{2}$ nanoparticles in water at different time delays after excitation at $400 \mathrm{~nm}$. 
and has been fitted with a multi-exponential function with the time constants of $\tau_{1}=800 \pm 70$ fs $(54.9 \%)$ $\tau_{2}=5.3 \pm 0.5 \mathrm{ps} \quad(26.4 \%) \quad \tau_{3}=50 \pm 2 \mathrm{ps} \quad(7.7 \%)>$ $400 \mathrm{ps}(11 \%)$. As we have attributed the $610 \mathrm{~nm}$ peak to the cation radical of TPP-cat, we expect that kinetic decay trace is similar to that of the conduction band electron at $900 \mathrm{~nm}$. However, we have observed that kinetic decay trace at $610 \mathrm{~nm}$ has been best fitted with a multi-exponential function with time constants of $\tau_{1}=130 \pm 50$ fs $(67 \%) \tau_{2}=800 \pm$ $70 \mathrm{fs}(14.6 \%) \tau_{3}=5.3 \pm 0.5 \mathrm{ps}(5.8 \%) \tau_{4}=50 \pm 2 \mathrm{ps}$ $(5.8 \%) \tau_{5}=>400$ ps $(6.8 \%)$. The extra fast component (130 fs) has not been observed in other wavelengths (other than 550-610 nm region).

Figure 9 shows the transient absorption spectra of TPP-cat $/ \mathrm{TiO}_{2}$ system after excitation at $800 \mathrm{~nm}$. The

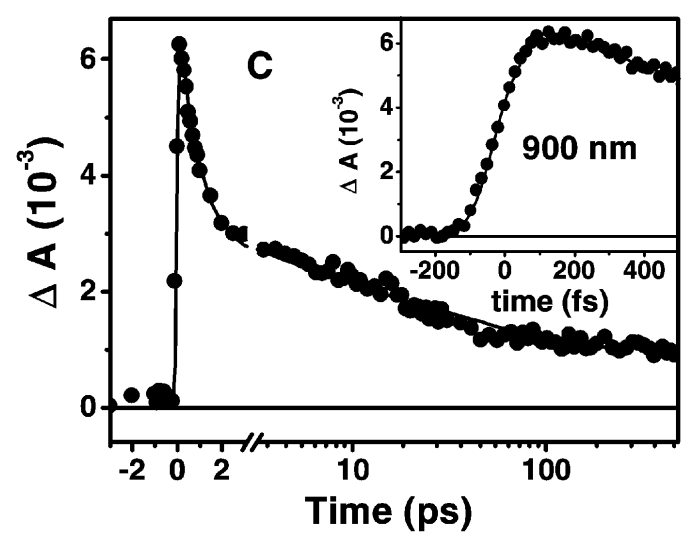

Figure 8. Kinetic decay trace of injected electrons in the conduction band at $900 \mathrm{~nm}$ in TPP-cat sensitized $\mathrm{TiO}_{2}$ nanoparticles (inset shows the kinetic trace at $900 \mathrm{~nm}$ at shorter time scale).

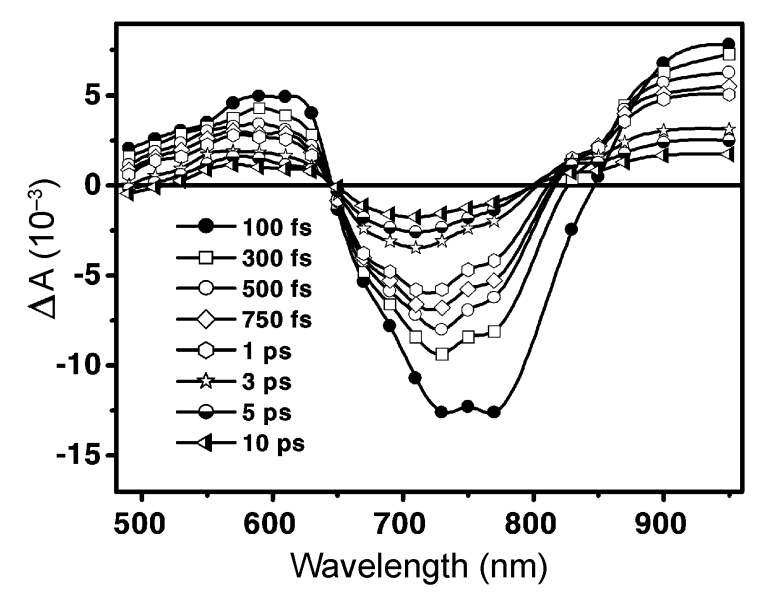

Figure 9. Transient absorption spectra of TPP-cat sensitized $\mathrm{TiO}_{2}$ nanoparticle in water at different time delays after excitation at $800 \mathrm{~nm}$. transient spectra show a transient absorption peak at $610 \mathrm{~nm}$, two bleach peaks with maxima at $770 \mathrm{~nm}$ and $730 \mathrm{~nm}$, and broad absorption band in the 800$1000 \mathrm{~nm}$ region. It is interesting to see that the bleach at $770 \mathrm{~nm}$ recovers much faster as compared to $730 \mathrm{~nm}$. We have also monitored the transient decay kinetics at $610 \mathrm{~nm}$ and $900 \mathrm{~nm}$. Figure 10 shows the decay/recovery kinetics at different wavelengths at shorter time domain (up to $5 \mathrm{ps}$ ). It is seen from Figure 10 that bleach at $770 \mathrm{~nm}$ recovers faster as compared to that at 730 and $670 \mathrm{~nm}$. However, it is interesting to see that decay dynamics at $900 \mathrm{~nm}$ matches fairly well with the recovery kinetics at $670 \mathrm{~nm}$. We have also monitored the decay/recovery

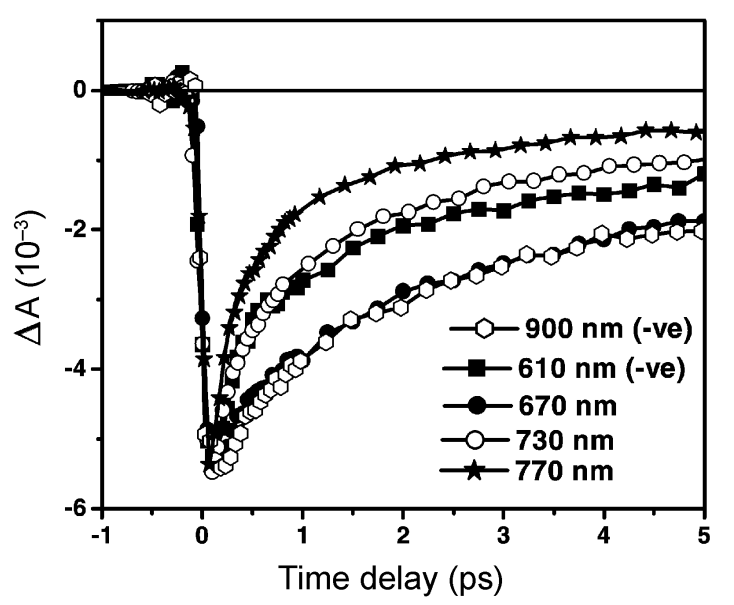

Figure 10. Kinetic decay traces at $610 \mathrm{~nm}$ and $900 \mathrm{~nm}$ and bleach recovery kinetics at $670 \mathrm{~nm}, 730 \mathrm{~nm}$ and $770 \mathrm{~nm}$ at shorter time scales (up to $5 \mathrm{ps}$ ) in TPP-cat sensitized $\mathrm{TiO}_{2}$ nanoparticles after excitation at $800 \mathrm{~nm}$.

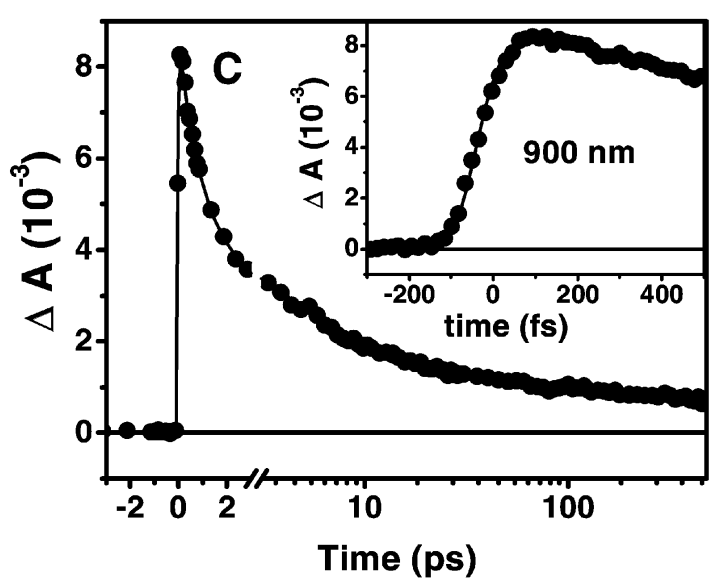

Figure 11. Kinetic decay trace of the injected electron in the conduction band at $900 \mathrm{~nm}$ in TPP-cat sensitized $\mathrm{TiO}_{2}$ nanoparticles (inset shows the kinetic trace at $900 \mathrm{~nm}$ at shorter time scale). 
Table 1. Excited state decay kinetics of TPP-cat sensitized $\mathrm{TiO}_{2}$ nanoparticle systems at different wavelengths after exciting at $400 \mathrm{~nm}$ and $800 \mathrm{~nm}$ laser light.

\begin{tabular}{lll}
\hline $\begin{array}{l}\text { Probe wavelength } \\
(\mathrm{nm})\end{array}$ & $\begin{array}{c}\text { Lifetimes with } 400 \mathrm{~nm} \\
\text { excitation* }\end{array}$ & $\begin{array}{c}\text { Lifetimes with } 800 \mathrm{~nm} \\
\text { excitation* }\end{array}$ \\
\hline 610 & $\tau_{1}=130 \pm 50(67)$ & $\tau_{1}=100 \pm 50(64)$ \\
& $\tau_{2}=800 \pm 70(14 \cdot 6)$ & $\tau_{2}=850 \pm 70(17 \cdot 7)$ \\
& $\tau_{3}=5 \cdot 3 \pm 0 \cdot 5(5 \cdot 8)$ & $\tau_{3}=5 \cdot 1 \pm 0 \cdot 5(12 \cdot 4)$ \\
& $\tau_{4}=50 \pm 2(5 \cdot 8)$ & $\tau_{4}=>400(5 \cdot 9)$ \\
& $\tau_{5}=>400(6 \cdot 8)$ & \\
710 & $\tau_{1}=800 \pm 70(54 \cdot 9)$ & $\tau_{1}=100 \pm 50(15 \cdot 5)$ \\
& $\tau_{2}=5 \cdot 1 \pm 0 \cdot 5(26 \cdot 4)$ & $\tau_{2}=850 \pm 70(38 \cdot 8)$ \\
& $\tau_{3}=50 \pm 2(7 \cdot 7)$ & $\tau_{3}=5 \cdot 3 \pm 0 \cdot 5(36)$ \\
& $\tau_{4}=>400(11)$ & $\tau_{4}=>400(9 \cdot 7)$ \\
& $\tau_{1}=800 \pm 70(57 \cdot 3)$ & $\tau_{1}=800 \pm 70(44)$ \\
& $\tau_{2}=5 \cdot 3 \pm 0 \cdot 5(14)$ & $\tau_{2}=5 \cdot 1 \pm 0.5(40 \cdot 7)$ \\
$\tau_{3}=50 \pm 2(15 \cdot 3)$ & $\tau_{3}=50 \pm 5(3 \cdot 3)$ \\
& $\tau_{4}=>400(13 \cdot 4)$ & $\tau_{4}=>400(12)$ \\
770 & & $\tau_{1}=100 \pm 50(63 \cdot 6)$ \\
& & $\tau_{2}=850 \pm 70(25 \cdot 6)$ \\
& & $\tau_{3}=5 \cdot 3 \pm 0.5(7 \cdot 8)$ \\
& & $\tau_{4}=>400(3)$ \\
\hline
\end{tabular}

*Values are in femtoseconds, and numbers in parentheses are percentages

kinetics (both short time and long time) at $900 \mathrm{~nm}$ and have shown in figure 11. It has been observed that the decay/recovery kinetics have been fit with a multi-exponential function with different time constants (table 1). The excited state decay kinetics at $610 \mathrm{~nm}$ has been best fitted with time constants of $\tau_{1}=100 \pm 50 \mathrm{fs}(64 \%) \tau_{2}=850 \pm 70 \mathrm{fs}(17.7 \%) \tau_{3}=$ $5 \cdot 1 \pm 0.5$ ps $(12.4 \%) \tau_{4}=>400$ ps $(5.9 \%)$. However the excited decay kinetics at $900 \mathrm{~nm}$ has been fit with time constants of $\tau_{1}=800 \pm 70 \mathrm{fs}(44 \%) \tau_{2}=$ $5.1 \pm 0.5 \mathrm{ps} \quad(40.7 \%) \quad \tau_{3}=50 \pm 5 \mathrm{ps} \quad(3.3 \%) \quad \tau_{4}=$ $>400$ ps $(12 \%)$. It is interesting to see that the ultrafast ( $\sim 100 \mathrm{fs}$ ) decay component at $900 \mathrm{~nm}$ is absent. First of all, we will discuss the generation process of $e^{-}$СB observed in near IR region. Kinetic decay trace monitored in that region $(900-1000 \mathrm{~nm})$ appears to have less interference from the other transient species. The processes involved in TPP-cat/ $\mathrm{TiO}_{2}$ system after excitation at $400 \mathrm{~nm}$ laser light are complicated as compared to $800 \mathrm{~nm}$ laser excitation. For the same reason, the excited state dynamics of TPP-cat $/ \mathrm{TiO}_{2}$ system after excitation at $800 \mathrm{~nm}$ has been discussed in advance. As mentioned earlier, a bleach at $770 \mathrm{~nm}$, which corresponds to the CT band of the dye with $\mathrm{TiO}_{2}$ nanoparticles and TPP-cat (Q-band) has been observed in the transient absorption spectra (figure 9) of TPP-cat $/ \mathrm{TiO}_{2}$ system after excitation at $800 \mathrm{~nm}$, which recovers very fast (table 1). It has also been observed that the time constant (100 fs component) for $610 \mathrm{~nm}$ decay kinetics match with the recovery kinetics at $770 \mathrm{~nm}$ (table 1). From the above observation, the excited state absorption band at $610 \mathrm{~nm}$ can be attributed to the excited CT band, which is a charge-separated state. The charge-separated state can return back to the ground state (path 1) or it can diffuse in to the conduction band (i.e. electron injection in the nanoparticles) (path 2) (scheme 2). The ultrafast bleach recovery at $770 \mathrm{~nm}$ and excited state decay kinetics at 610 can be ascribed to path 1 . It is interesting to observe that the bleach at $770 \mathrm{~nm}$ at $\sim 3 \mathrm{ps}$ (figure 9) merges with the $710 \mathrm{~nm}$ bleach. Transient measurements (table 1) have shown that the lifetime of excited CT state is as short as $\sim 100 \mathrm{fs}$. However, it is striking to see that the transient absorption at $610 \mathrm{~nm}$ persists even after $3 \mathrm{ps}$. This is because of the fact that the transient absorption spectra of the excited CT band and cation radical can be identical. ${ }^{18}$ The observation of excited CT state in dyesensitized semiconductor nanoparticles seems interesting, however, this kind of observation has also been made earlier. The presence of excited CT state has been observed by Furube et $a l^{19}$ in coumarin dye (NKX-2311) sensitized $\mathrm{ZnO}$ nanoparticles. As a result, upon excitation of the CT band of complex 2 by $800 \mathrm{~nm}$ laser light we can expect to see the excited 


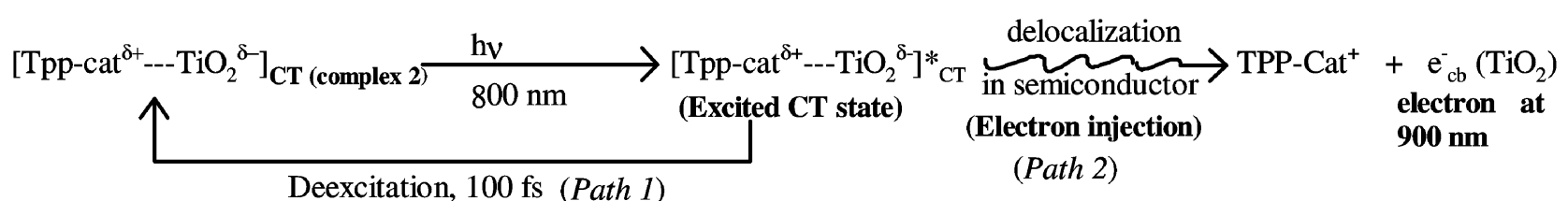

Chart 1.
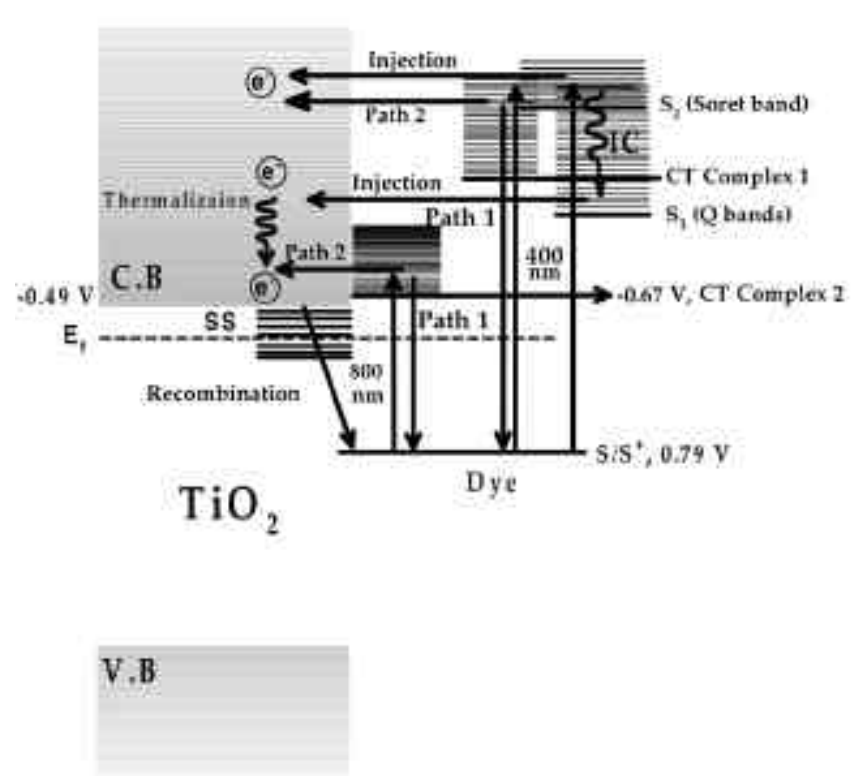

Scheme 2. Mechanistic scheme showing the various processes involved in the electron-transfer dynamics of TPP-cat sensitized $\mathrm{TiO}_{2}$ nanoparticles after excitation at 400 and $800 \mathrm{~nm}$.

CT state, which is an intermediate state prior to electron injection. As the density of states in the conduction band of $\mathrm{TiO}_{2}$ is much higher, so one could expect efficient electron injection into the conduction band of $\mathrm{TiO}_{2}$ nanoparticles (path 2). However, we have observed another de-excitation channel of excited CT state to the ground state (path $1)$. The above process can be schematically represented as in chart 1 .

Now, let us discuss the optical processes occurring in TPP-cat $/ \mathrm{TiO}_{2}$ system on excitation at $400 \mathrm{~nm}$. It is quite clear from figure 1 that both Soret band $\left(S_{2}\right)$ and complex 1 have absorption at $400 \mathrm{~nm}$. So on $400 \mathrm{~nm}$ laser excitation both the bands are excited. Energetically, Soret band is much higher as compared to that of the bottom of the conduction band edge, so thermodynamically electron injection is possible. It has been observed in porphyrin molecules that de-excitation process from Soret band $\left(S_{2}\right)$ to $Q$ band $\left(S_{1}\right)$ can be as fast as $<10 \mathrm{fs}^{20}$ through internal conversion (IC). So the population to $Q$ band is also possible, whose energy level is also higher than conduction band edge of $\mathrm{TiO}_{2}$ (scheme 2). Hence, electron injection is also possible from $Q$ band. The above process can be schematically represented in chart 2 .

However, electron injection would take place only from Soret and $Q$ band, and ET dynamics would have been much simpler. Decay kinetics at $610 \mathrm{~nm}$ (for the cation radical) would have matched with the decay kinetics at $900 \mathrm{~nm}$ (for $e_{\mathrm{CB}}^{-}$) and bleach recovery kinetics at $710 \mathrm{~nm}$. It is seen in table 1 that decay kinetics at $900 \mathrm{~nm}$ matches with bleach recovery kinetics at $710 \mathrm{~nm}$. However, it has been observed that decay kinetics at $610 \mathrm{~nm}$ is fitted with a multi-exponential function with an extra $\approx 100 \mathrm{fs}$ time constant (table 1). Thus the excited state dynamics in TPP-cat/ $\mathrm{TiO}_{2}$ is complicated at $400 \mathrm{~nm}$ excitation as compared to $800 \mathrm{~nm}$ excitation. On exciting complex 1 initially the hole get localized on catechol moiety which a part of TPP-cat, so it is quite obvious that the hole migrates very fast $(<10 \mathrm{fs})$ to the porphyrin moiety via electron transfer from TPP. As our instrument response $\approx 100 \mathrm{fs}$, it is difficult to monitor the molecular process which takes place sub-10 fs. As a result, on excitation of complex 1, we did not observe any signature of bleach in the 400-600 nm region. The above photochemical process can be schematically represented as in chart 3 .

Now from chart 2 we can explain the excitation process of complex 1 by $400 \mathrm{~nm}$ excitation, the electron being localized on the $\mathrm{Ti}$ atom, resulting a charge-separated state. Here also the localized electron can recombine very fast $(\approx 130 \mathrm{fs})$ with the parent TPP-cat cation (path 1) or it can diffuse in to the conduction band (path 2). The ultrafast component of the excited state decay kinetics at 610 can be ascribed to path 1.

Nonetheless, BET dynamics can be measured following bleach recovery kinetics at $710 \mathrm{~nm}$ or elec- 


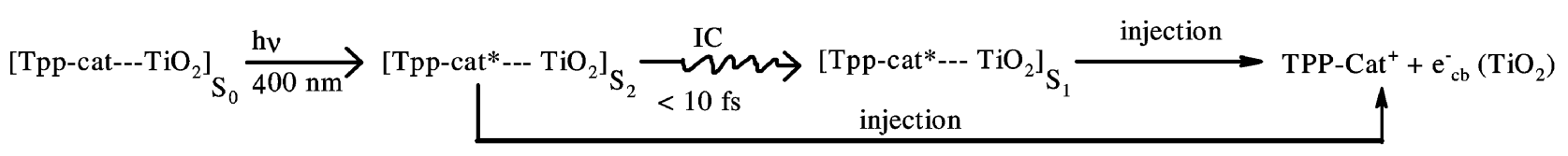

Chart 2.

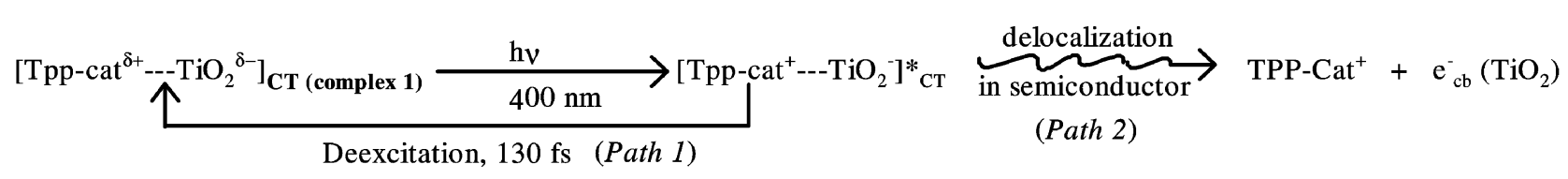

Chart 3.

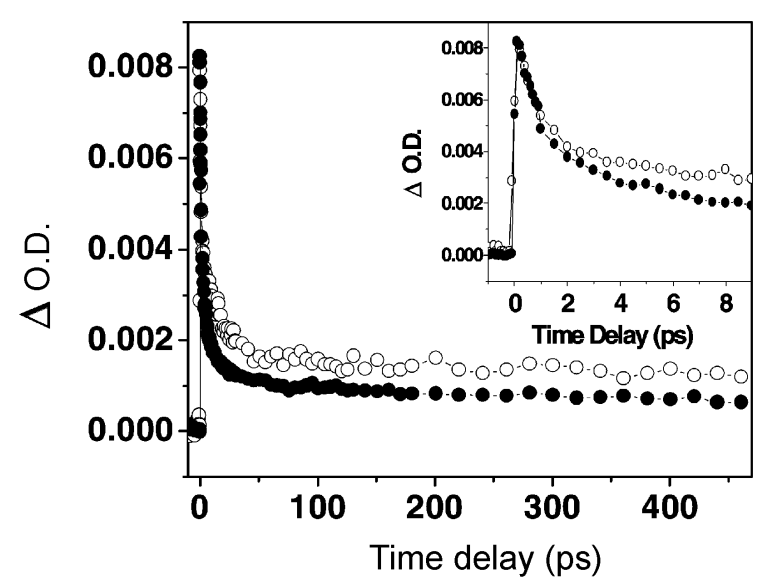

Figure 12. Comparison of charge recombination (BET) dynamics in TPP-cat sensitized $\mathrm{TiO}_{2}$ nanoparticles monitoring injected electrons at $900 \mathrm{~nm}$ exciting $400 \mathrm{~nm}(\mathrm{O})$ and $800 \mathrm{~nm}(\bullet)$ laser light.

tron in the conduction band at $900 \mathrm{~nm}$ (table 1). One of the main aims of this investigation is to study interfacial ET dynamics exciting different bands in TPP-cat $/ \mathrm{TiO}_{2}$ system exciting $400 \mathrm{~nm}$ and $800 \mathrm{~nm}$ laser light. Figure 12 shows the comparison of BET dynamics in TPP-cat $/ \mathrm{TiO}_{2}$ system exciting $400 \mathrm{~nm}$ and $800 \mathrm{~nm}$ laser light after monitoring the signal of conduction band electron at $900 \mathrm{~nm}$. It is interesting to see that the recombination dynamics is little faster in $\mathrm{TPP}-\mathrm{cat} / \mathrm{TiO}_{2}$ system at $800 \mathrm{~nm}$ excitation as compared to that of at $400 \mathrm{~nm}$ excitation. This may be due to the fact that at $800 \mathrm{~nm}$ light only excites complex 2, where de-excitation to the ground state dominates BET dynamics due to strong coupling. On the other hand, $400 \mathrm{~nm}$ light excites both the Soret band and complex 1 . On exciting the complex 1 , both electron injection and BET process can be faster, as we have observed on exciting complex 2. However, on exciting on the Soret band, electron injection can take place from both Soret and $Q$ band (scheme 2). In that case, electron injection takes place through the excited state where BET dynamics is slower as compared to the complex excitation process. As a result, the net effect is that at $400 \mathrm{~nm}$ excitation, BET dynamics is slower as compared to that at $800 \mathrm{~nm}$ excitation.

\section{Conclusion}

Ru-cat and TPP-cat couple strongly with $\mathrm{TiO}_{2}$ nanoparticles as the dye is absorbed on the nanoparticle surface through the pendant catechol moiety. As the $\mathrm{p} K_{a}$ values for these catecholate sensitizers are as high as $>9.5$, this particular dye-nanoparticle system gives greater stability even at higher $\mathrm{pH}$ conditions unlike other carboxylate compounds. We have studied interfacial ET dynamics in Ru-cat/ $/ \mathrm{TiO}_{2}$ and TPP-cat systems using femtosecond transient absorption measurements. In $\mathrm{Ru}-\mathrm{cat} / \mathrm{TiO}{ }_{2}$ system electron injection is found to be single exponential and pulse-width limited ( $<100 \mathrm{fs})$. In the present investigation electron injection process competes with thermalization process in the photo-excited state of Ru-cat. Our results indicate that electron injection take place predominantly from non-thermalized singlet state ( $\left.{ }^{1} \mathrm{MLCT}\right)$, and/or non-thermalized triplet state $\left({ }^{3} \mathrm{MLCT}\right)$ or a combination of both, which is a unique observation in ET dynamics in Ru-polypyridyl/ $/ \mathrm{TiO}_{2}$ systems studied so far. Strong coupling in dye-nanoparticle system facilitates ultrafast single-exponential electron injection, which competes with the thermalization process of excited states. 
BET dynamics in Ru-cat/Tio2 system have been found to be multi-exponential process with time constants of $1.5 \mathrm{ps}(34.8 \%), 70 \mathrm{ps}(26.8 \%)$, and 400 ps $(38.4 \%)$.

ET dynamics in TPP-cat/ $/ \mathrm{TiO}_{2}$ system is complicated. Reaction channel (path) for ET processes is different for different excitation wavelengths $(400 \mathrm{~nm}$ and $800 \mathrm{~nm}$ ). Our studies revealed that $800 \mathrm{~nm}$ excitation populate the excited CT state (complex 2). After that it follows two different paths. Path 1 is the photo-excited CT state which gets de-excited to the ground state with time constant $\approx 100 \mathrm{fs}$. Path 2 is from photo-excited CT state diffusion of electrons and takes place into the conduction band of the nanoparticles, which is electron injection. On the other hand, $400 \mathrm{~nm}$ excitation populates the $S_{2}$ state (Soret band) of the porphyrin and it can relax very fast and populates the $S_{1}$ state ( $Q$-band) and electron injection can take place from both the states. However, $400 \mathrm{~nm}$ laser light also excites complex 1 where injected electrons in the $\mathrm{Ti}$ atom recombine very fast with the parent cation with a time scale of $\approx 130$ fs (path 1) and also diffuse in the conduction band of $\mathrm{TiO}_{2}$ (path 2). We have also observed that BET dynamics is multi-exponential in TPP-cat $/ \mathrm{TiO}_{2}$ system is faster at $800 \mathrm{~nm}$ light excitation as compared to that at $400 \mathrm{~nm}$ light excitation.

\section{Acknowledgment}

I would like to thank my co-workers Drs G Ramakrishna, D K Palit and Amitava Das (CSMCRI, Bhavnagar) for fruitful discussions. I would also like to thank Dr S K Sarkar of our Division and Dr T Mukherjee, Chemistry Group for their encouragement.

\section{References}

1. Pelizzetti M and Schiavello M 1997 Photochemical conversion and storage of solar energy (Dordrecht: Kluwer)
2. O’Regan B and Grätzel M 1991 Nature (London) 353 737

3. Nazeeruddin M K, Pechy P, Renouard T, Zakeeruddin S M, Humphry-Baker R, Comte P, liska P, Cevey L, Costa E, Shklover V, Spiccia L, Deacon G B, Bignozzi C A and Gratzel M $2001 \mathrm{~J}$. Am. Chem. Soc. 1231613

4. Nazeeruddin M K, Zakeeruddin S M, HumphryBaker R, Jirousek M, Liska P, Vlachopoulus N, Skhlover V, Fischer C H and Gratzel M 1999 Inorg. Chem. 386298

5. Rego G and Batista V S 2003 J. Am. Chem. Soc. 125 7989

6. Ramakrishna G, Ghosh H N, Singh A K, Palit D K and Mittal J P 2001 J. Phys. Chem. B105 12786

7. Ramakrishna G, Singh A K, Palit D K and Ghosh H N 2004 J. Phys. Chem. B108 1701

8. Shukla A D, Whittl B, Bajaj H C, Das A and Ward M D 1999 Inorg. Chim. Acta 28589

9. Jose A D, Shukla A D, Krishnakumar D, Ganguly B, Das A, Ramakrishna G, Palit D K and Ghosh H N 2005 Inorg Chem. 442414

10. Hagfeldt A and Gratzel M 1995 Chem. Rev. 9549

11. Ramakrishna G, Jose A D, Krishnakumar D, Das A, Palit D K and Ghosh H N 2005 J. Phys. Chem. B109 15445

12. Ramakrishna G, Jose A D, Krishnakumar D, Das A, Palit D K and Ghosh H N 2006 J. Phys. Chem. B110 10197

13. Asbury J B, Hao E, Wang Y Q, Ghosh H N and Lian T 2001 J. Phys. Chem. B105 4545

14. Benko G, Kallioinen J, Korppi-Tommola J E I, Yartsev A P and Sundstrom V $2002 \mathrm{~J}$. Am. Chem. Soc. 104489

15. Bhasikuttan A C, Suzuki M, Nakashima S and Okada T 2002 J. Am. Chem. Soc. 1048398

16. Lee H Y, Kim Y, Dutta P K and Das A 2003 Inorg. Chem. 424215

17. Bonnett R, Lambert C, Land E J, Scourides P A, Sinclair R S and Truscott T G 1983 Photochem. Photobiol. 381

18. Mataga N 1991 in Electron transfer in inorganic, organic, and biological systems (eds) J R Bolton, N Mataga and $G$ McLendon (Advances in Chemistry Series) (Washington, DC:) pp. 91-115

19. Furube A, Katoh R, Yoshihara T, Hara K, Murata S, Arakawa H and Tachiya M 2004 J. Phys. Chem. B108 12583

20. Zhong Q, Wang Z, Liu Y, Zhu Q and Kong F 1996 J. Chem. Phys. 1055377 